\title{
Advanced diabetic kidney disease complicated by acute interstitial nephritis: A case report and literature review
}

\author{
JIN HE, SHUBEI CHEN and WEIHONG HUANG
}

Division of Nephrology and Endocrinology, Chonggang General Hospital, Chongqing 400016, P.R. China

Received February 25, 2021; Accepted May 18, 2021

DOI: $10.3892 /$ wasj.2021.108

\begin{abstract}
Currently, diabetic kidney disease (DKD) is the main cause of chronic kidney disease (CKD) and end-stage renal disease (ESRD) worldwide. The condition of advanced DKD is severe and is often accompanied by a number of complications, such as heart failure and pulmonary infection, requiring multiple-drug treatment. However, any type of medicine has the potential to cause acute interstitial nephritis (AIN). In addition, as renal fibrosis in advanced DKD is already severe without taking any complications, such as AIN into account, it remains unclear whether steroidal therapy can benefit the condition. The present study describes the case of a patient with advanced DKD complicated by AIN, who was administered steroidal therapy. Ultimately, the patient's renal function partially improved. The present study also presents a brief review, discussing past experience on DKD with complications. On the whole, it is demonstrated that even patients with advanced DKD may still benefit from steroid therapy.
\end{abstract}

\section{Introduction}

As the number of patients worldwide with type 2 diabetes rapidly is increasing, diabetic kidney disease (DKD) has become the leading cause of chronic kidney disease (CKD) and end-stage renal disease (ESRD) (1-3). Acute interstitial nephritis (AIN) is one of the main causes of acute kidney injury (AKI) and affects $\sim 15-27 \%$ of patients with unexplained AKI. AIN will eventually develop into CKD, or even end-stage renal disease (ESRD), without timely and appropriate treatment (4-6).

Although DKD and AIN are clinically common, to the best of our knowledge, there is no report available to date of advanced DKD complicated by AIN. In particular, there is a lack of relevant experience with the use of steroids for these patients. The present study reports a case of a patient with

Correspondence to: Dr Jin He, Division of Nephrology and Endocrinology, Chonggang General Hospital, Chongqing 400016, P.R. China

E-mail: hejin19811118@sina.com

Key words: diabetic kidney disease, acute interstitial nephritis, steroid, chronic kidney disease, end-stage renal disease advanced DKD complicated by AIN treated with steroids. The patient's renal function was partially recovered following steroid therapy.

\section{Case report}

A 62-year-old Chinese male was admitted to the Department of Cardiology, Chonggang General Hospital (Chongqing, China) with a 1-day episode of dyspnea and orthopnea. A physical examination revealed a body temperature of $36.5^{\circ} \mathrm{C}$, a breathing rate of 18 breaths $/ \mathrm{min}$, a heart rate of 104 beats $/ \mathrm{min}$ and a blood pressure of 160/86 $\mathrm{mmHg}$. There were moist and dry rales in both lungs, and he had edema of the lower limbs. A CT scan revealed bilateral pleural effusion and patchy opacity shadows of the bilateral lungs (Fig. 1A and B). Color Doppler echocardiography revealed left and right atrium dilation (Fig. 1C and D) and an electrocardiogram revealed sinus tachycardia (Fig. 1E). The laboratory test findings for the time of admission are presented in Table I. The patient provided informed consent for his involvement in the present study.

The patient had a 6-year history of hypertension (of which the highest blood pressure value was $180 / 100 \mathrm{mmHg}$ ), a 10 -year history of diabetes, and a 1-year history of diabetic retinopathy. At the time of admission, the patient was clinically diagnosed with the following: i) Grade 3 hypertension (severe), hypertensive heart disease, heart enlargement and heart failure (cardiac function grade III-IV); ii) type 2 diabetes, diabetic retinopathy, diabetic kidney disease, CKD stage 5 and renal anemia; and iii) pneumonia.

Following treatment with felodipine ( $5 \mathrm{mg}$, once a day), recombinant human brain natriuretic peptide (rhBNP, $0.01 \mu \mathrm{g} / \mathrm{kg} / \mathrm{min}$ for 3 days), furosemide (100 $\mathrm{mg}$, once a day for 7 days) and antibiotics (cefuroxime, $0.75 \mathrm{~g}$, every $12 \mathrm{~h}$ for 10 days), the patient's dyspnea improved and his lower limb edema gradually disappeared. On the 8th day following admission, the patient once again had dyspnea, and the urine output decreased progressively. Despite increasing the furosemide dosage, the patient's symptoms did not improve significantly, and his renal function progressively deteriorated. On day 10, the patient was transferred to the Division of Nephrology and Endocrinology, Chonggang General Hospital for hemodialysis treatment.

Following hemodialysis, the patient's dyspnea and systemic edema were relieved. A routine laboratory examination (at day 13 after admission) revealed that the patient's eosinophil 
count was markedly higher than that at admission, and there was no evidence of deterioration of renal function due to autoimmune diseases (Table I). Considering that the patient presented with a typical clinical course of AKI, and considering the patient's history of antibiotics use and the increased eosinophil count, and despite the fact that the patient did not have a fever, joint pain, or rash during the course of the disease, AIN was suspected.

After obtaining the consent of the patient, a kidney biopsy was performed and the renal biopsy specimens were sent to the Guangzhou Kingmed Center For Clinical Laboratory, Co., Ltd. for staining. The kidney biopsy findings were as follows: Upon light microscopy, the biopsy specimen revealed 24 glomeruli, 11 of which displayed glomerular sclerosis, and nodular hyperplasia in the glomerular mesangial area was shown in the rest; hyperplasia was mainly in the mesangial matrix and lobulated capillary loops were observed. Kimmelstiel-Wilson lesions (K-W lesions) were also visible, and there were some extensive capillary hemangiomas. There was diffuse atrophy of the renal tubules (the atrophic area accounted for $\sim 80 \%$ ) and diffuse infiltration of inflammatory cells into the renal interstitium with fibrosis (Fig. 2A-D). The immune complex was not shown on immunofluorescence microscopy (Fig. 2E and F). Electron microscopy revealed the partial atrophy of the renal tubules. Inflammatory cell infiltration into the renal interstitium was accompanied by the proliferation of collagen fibers (Fig. 2G and H). The diagnosis made was 'nodular diabetic glomerulosclerosis complicated by interstitial nephritis'.

Considering that the patient had been in a state of continuous oliguria, and there was no improvement trend, it was decided that he should receive prednisone treatment, even though the renal biopsy revealed severe fibrosis. With the consent of the patient, prednisone was administered at $30 \mathrm{mg} /$ day $(0.5 \mathrm{mg} / \mathrm{kg})$ for 14 days. After 2 weeks, the dosage was reduced to $20 \mathrm{mg} /$ day for 7 days; after 1 week, the dosage was reduced to $10 \mathrm{mg} /$ day for 7 days; the drug was then terminated. Following prednisone treatment for $24 \mathrm{~h}$, the patient's urine volume significantly improved. On the 12th day following treatment, the 24-h urine volume reached 1,900 ml/day (without diuretics) (Fig. 3), and his kidney function also partially improved (Scr, $7.56 \mathrm{mg} / \mathrm{dl}$; eGFR, $7.41 \mathrm{ml} / \mathrm{min} / 1.73 \mathrm{~m}^{2}$ ).

\section{Discussion}

AIN is characterized by renal interstitial inflammatory cell filtration and interstitial edema accompanied by the rapid deterioration of renal function. If treatment is not administered in a timely manner, it can gradually progress to CKD or even ESRD.

There are a number of causes of AIN that can be roughly divided into drug-induced, infection-related, idiopathic and systemic disease (7). Among these pathogenic factors, drugs are the leading cause of AIN. In two clinical studies involving 121 patients with AIN, drug-induced AIN (DI-AIN) accounted for the majority $(91 \%)$ of cases $(8,9)$.

In theory, any medicine or even transdermal patch may cause AIN, and antibiotics, non-steroidal anti-inflammatory drugs, and proton pump inhibitors are the most frequent offenders $(6,10,11)$. Typical DI-AIN may present as a triad of fever, rash and eosinophilia; however, these three characteristics co-occur in only $10-15 \%$ of patients with DI-AIN, and DI-AIN may present weeks or months following the use of the drug (6). Thus, it is very difficult to diagnose DI-AIN promptly and accurately. In the case presented herein, the patient had no fever or rash and only presented a rapid deterioration of renal function and an increase in the blood eosinophil count. The patient had a clear history of diabetes and diabetic retinopathy, and the renal function had reached CKD stage 5 upon admission. Thus, it would be easy for a non-nephrology specialist to attribute the deterioration of renal function to the aggravation of DKD or cardiorenal syndrome caused by heart failure $(12,13)$. However, following the careful analysis of the course of the disease, it was evident that the patient presented a typical course of AKI. Combined with the history of antibiotic use and the increased eosinophil count, the association between DI-AIN with the rapid deterioration in renal function was fairly straightforward.

The efficacy of using steroids in the treatment of DI-AIN was once controversial. A previous retrospective study on 60 patients with AIN (drug-related in $92 \%$ of cases), who were confirmed by renal biopsy, compared the effects of steroidal and conservative treatment on renal function; the conclusion of that study was that there was no significant difference in serum creatinine levels between the two groups following 12 months of treatment (8). However, in another multicenter retrospective study including 61 patients with biopsy-proven DI-AIN, the final serum creatinine levels were significantly lower in the corticosteroid-treated groups, while almost half of the conservatively managed patients remained on chronic dialysis; further analysis revealed that when steroid treatment was delayed (by an average of 34 days), renal function did not return to baseline levels compared with those who received steroid treatment within the first 2 weeks after the causative drug was withdrawn (9). It is currently considered that, for AIN, the early and limited use of low-dose steroid therapy may alleviate $>85 \%$ of the patients' conditions without severe side-effects (14).

In the case presented herein, it was difficult to determine whether steroids should be for this patient. On the one hand, the patient suffered from diabetes, and the use of steroids may have aggravated the patient's diabetic condition; on the other hand, the patient's biopsy indicated that his kidneys had undergone severe fibrosis, and steroids may not significantly improve renal function. However, following an assessment of all the clinical evidence, it was decided that steroidal therapy would be initiated. Fortunately, during the treatment, the patient's blood sugar levels were well-controlled, no infections occurred, and the patient's renal function partially recovered.

The patient's renal function did not fully return to the level prior to admission. It was hypothesized that the following two reasons may be responsible for this phenomenon: The basic renal disease of this patient had been very severe, and following this attack, his renal disease further deteriorated; there was also an interval of 18 days from when the patient developed AKI to the initiation of steroid therapy. Previous research has suggested that renal interstitial fibrosis can occur after only 7 days of renal interstitial inflammation (15).

Residual renal function (RKF) is crucial for the prognosis of patients on dialysis, and a higher residual glomerular 
Table I. Laboratory data at admission and on the 13th and 38th day after admission.

\begin{tabular}{|c|c|c|c|}
\hline Parameter & Upon admission & Day 13 after admission & Day 38 after admission \\
\hline \multicolumn{4}{|l|}{ Complete blood count } \\
\hline Hemoglobin, g/dl & 9.0 & 8.1 & 11.3 \\
\hline White blood cell count, $/ \mathrm{mm}^{3}$ & $7.05 \times 10^{3}$ & $7.20 \times 10^{3}$ & $8.33 \times 10^{3}$ \\
\hline Neutrophil count, $\%$ & 68.3 & 57.9 & 58.5 \\
\hline Eosinophil count, \% (/mm $\left.{ }^{3}\right)$ & $4.3\left(0.30 \times 10^{3}\right)$ & $8.8\left(0.63 \times 10^{3}\right.$ & $3.2\left(0.5 \times 10^{3}\right.$ \\
\hline Platelet count, $/ \mathrm{mm}^{3}$ & $173 \times 10^{3}$ & $194 \times 10^{3}$ & $185 \times 10^{3}$ \\
\hline \multicolumn{4}{|l|}{ Blood chemistry tests } \\
\hline Alanine aminotransaminase, IU/1 & 17 & 16 & \\
\hline Aspartate aminotransferase, IU/1 & 14 & 13 & \\
\hline Total protein, $\mathrm{g} / \mathrm{l}$ & 69.4 & 64.1 & \\
\hline Albumin, $\mathrm{g} / \mathrm{l}$ & 38.4 & 31.3 & \\
\hline Total cholesterol, mmol/1 & 3.80 & 3.58 & \\
\hline Low-density lipoprotein cholesterol, mmol/1 & 2.21 & 1.76 & \\
\hline High-density lipoprotein cholesterol, mmol/1 & 0.67 & 1.26 & \\
\hline Triglyceride, $\mathrm{mmol} / \mathrm{l}$ & 1.65 & 1.30 & \\
\hline Glucose, $\mathrm{mmol} / \mathrm{l}$ & 6.2 & 3.1 & \\
\hline Glycosylated hemoglobin, $\%$ & 4.64 & & \\
\hline NT-proBNP, pg/ml & 10229.7 & 10883.0 & \\
\hline Scr, mg/dl & 4.94 & 9.57 & 7.56 \\
\hline $\mathrm{eGFR}, \mathrm{ml} / \mathrm{min} / 1.73 \mathrm{~m}^{2}$ & 12.73 & 5.94 & 7.41 \\
\hline \multicolumn{4}{|l|}{ Urinalysis } \\
\hline $\mathrm{pH}$ & 5.5 & 5.5 & \\
\hline Protein & $(+++)$ & $(++)$ & \\
\hline Blood & $(+)$ & $(+)$ & \\
\hline Glucose & $(+)$ & $(++)$ & \\
\hline Red blood cells & 2/HPF & 14/HPF & \\
\hline White blood cells & 0/HPF & 0/HPF & \\
\hline Urine protein to creatine ratio & $1647 \mathrm{mg} / \mathrm{g}$ creatinine & $1616 \mathrm{mg} / \mathrm{g}$ creatinine & \\
\hline \multicolumn{4}{|l|}{ Serum immunological factors } \\
\hline C3 & & $1.0 \mathrm{~g} / 1$ & \\
\hline $\mathrm{C} 4$ & & $0.3 \mathrm{~g} / 1$ & \\
\hline Myeloperoxidase-ANCA & & Negative & \\
\hline Proteinase 3-ANCA & & Negative & \\
\hline Anti-GBM antibody & & Negative & \\
\hline Anti-nuclear antibody & & Negative & \\
\hline Anti-dsDNA antibody & & Negative & \\
\hline Anti-SM antibody & & Negative & \\
\hline
\end{tabular}

NT-proBNP, N-terminal fragment of the brain natriuretic peptide precursor; eGFR, estimated glomerular filtration rate; Scr, serum creatinine; ANCA, antineutrophil cytoplasmic antibodies; GBM, glomerular basement membrane; dsDNA, double-stranded DNA; anti-SM, anti-Smith antibody.

filtration rate (rGFR) indicates a lower morbidity and mortality, and a higher quality of life for patients on peritoneal dialysis and hemodialysis (16). The NECOSAD study demonstrated that the mortality of patients on peritoneal dialysis decreased by $12 \%$ when the rGRE increased by $1 \mathrm{ml} / \mathrm{min} / 1.73 \mathrm{~m}^{2}$ (17). The CANUSA study revealed that, for every $250-\mathrm{ml}$ increase in urine volume (UV) in patients on peritoneal dialysis, the relative risk of mortality was reduced by $36 \%$ (18). Another study on patients on hemodialysis found that those with substantial RKF (>3.0 ml/min/1.73 $\mathrm{m}^{2}$ or UV >600 ml/day) had a significantly lower mortality rate and an improved quality of life than patients with a lower RKF $\left(\leq 3.0 \mathrm{ml} / \mathrm{min} / 1.73 \mathrm{~m}^{2}\right.$ or $\mathrm{UV} \leq 600 \mathrm{ml} /$ day) in the twice weekly hemodialysis regimen. In addition, there was no significant difference in first-year mortality between patients with substantial RKF who selected to undergo hemodialysis regimens twice a week and those who selected conventional hemodialysis regimens (thrice weekly) (19). In a previous study on patients undergoing new 

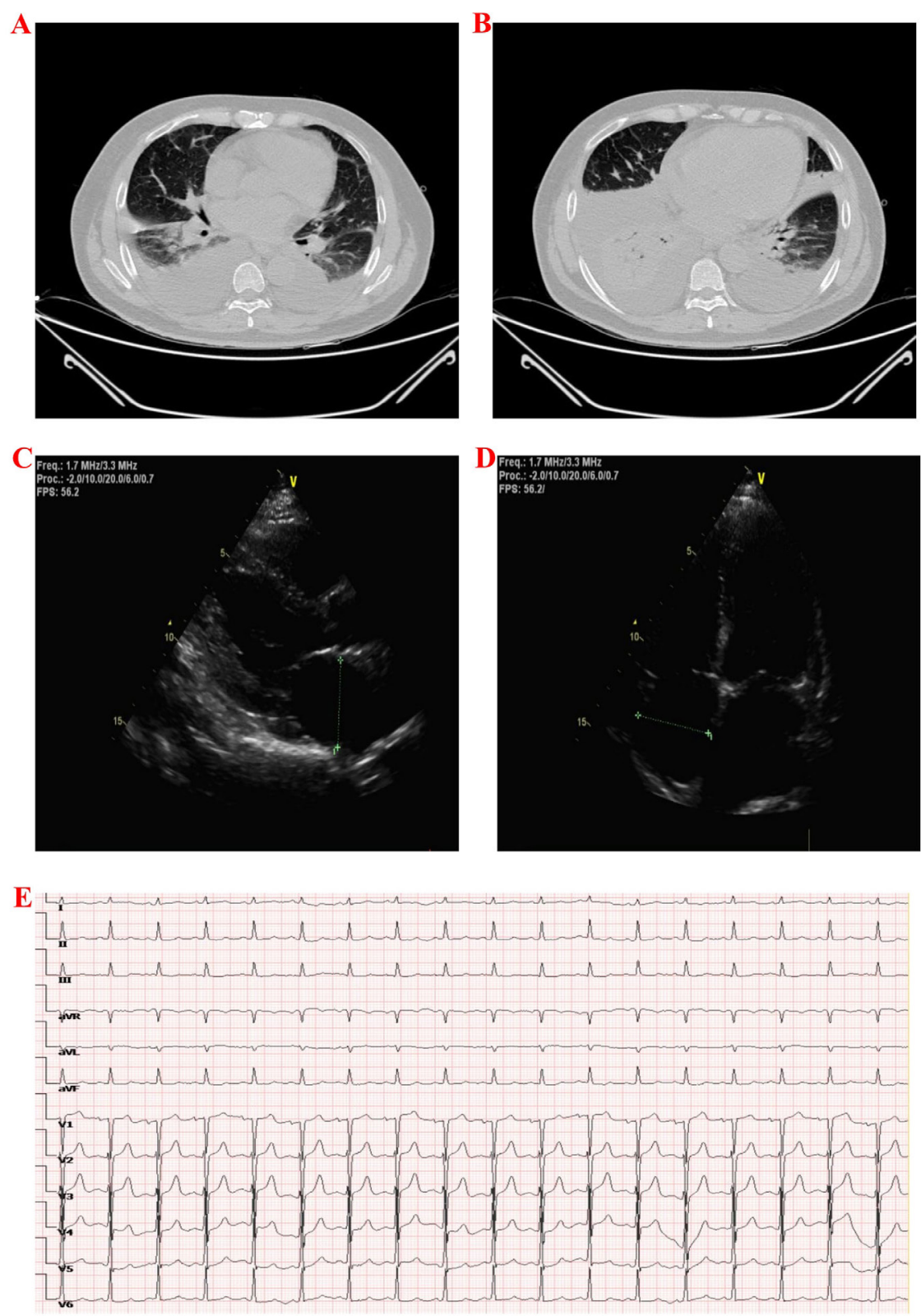

Figure 1. CT, Color Doppler echocardiography and electrocardiogram images. (A and B) CT scan revealed bilateral pleural effusion and patchy opacity shadows of bilateral lungs. (C and D) Color Doppler echocardiography showed left and right atrium dilation. (E) Electrocardiogram revealed sinus tachycardia.

onset long-term dialysis, when the RKF was retained after 1 year of dialysis, the mortality rate was reduced by $31 \%$ compared with that of patients with complete loss of renal function (20). In view of this finding, although the patient presented herein still needed to receive hemodialysis maintenance treatment, it was considered that the undertaken approach was warranted as renal function was partially successfully restored, which may improve the long-term prognosis of the patient.

DKD complicated by AIN is not, in fact, uncommon. A study on 101 patients with type 2 diabetes presenting with nephrotic syndrome, progressive deterioration of renal function, or active urinary sediment demonstrated that $11.9 \%$ of patients had DKD complicated by AIN (21). However, to date, at least to the best of our knowledge, there no clinical reports on advanced DKD complicated by AIN. It is considered that there are two potential reasons for this: Advanced DKD is a serious disease, often accompanied by a variety of complications, such as heart failure and pulmonary infection; these complications can, in turn, lead to the progressive deterioration of renal function. AIN, in addition to the deterioration of renal function, often lacks characteristic clinical manifestations; therefore, it is very difficult to identify such patients in clinical practice. Furthermore, renal fibrosis in patients with advanced DKD can be very severe, and the kidney functions of patients often deteriorate to the point to at which dialysis is required. Whether steroid therapy can improve the prognosis of these patients is unclear, and steroid therapy may also be associated with added risks of blood glucose fluctuation, infection and osteoporosis. Therefore, for such patients, a number of clinicians prefer to directly initiate dialysis treatment to avoid the adverse effects of steroids therapy. The experience acquired with the case in the present study suggests that steroid therapy may still improve the renal function of patients with advanced DKD complicated by AIN. 

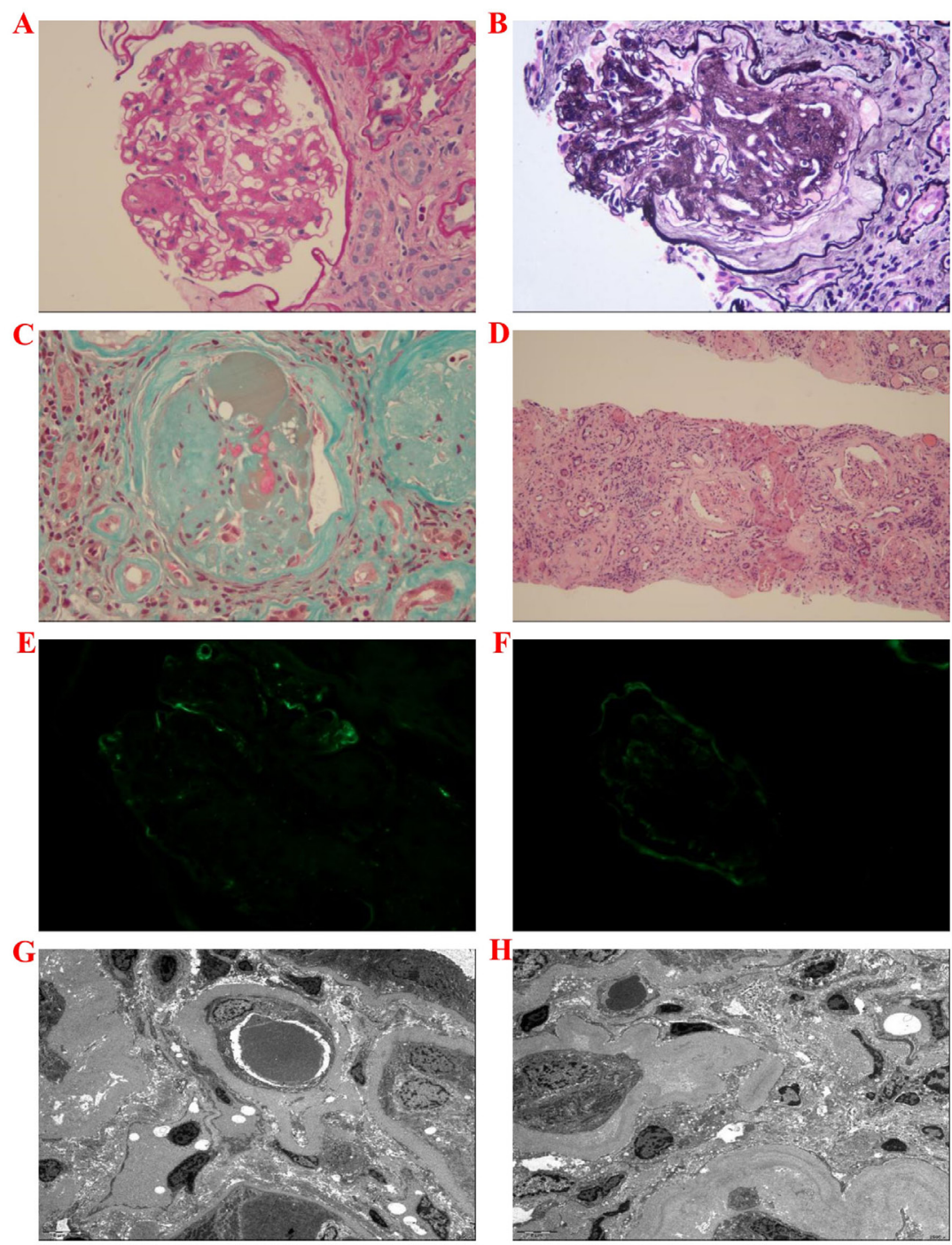

Figure 2. Kidney biopsy findings. (A and B) Under a light microscope, the glomerular mesangium exhibited nodular hyperplasia (mainly mesangial matrix hyperplasia), capillary loops with lobulated changes and Kimmelstiel-Wilson lesions (K-W lesions) could be observed. (C) Masson's staining revealed that 11 glomeruli (24 glomeruli in total) exhibited global glomerulosclerosis, and the renal interstitium exhibited severe fibrosis. (D) Diffuse inflammatory cell infiltration in the renal interstitium. (E and F) Immunofluorescence indicated no immune complex deposition. (G and H) Electron microscopy revealed partial renal tubule atrophy. Inflammatory cell infiltration in renal interstitium was accompanied by a proliferation of collagen fibers. PAS, periodic acid Schiff staining; PASM, periodic Schiff-methenamine silver staining; H\&E, hematoxylin and eosin.

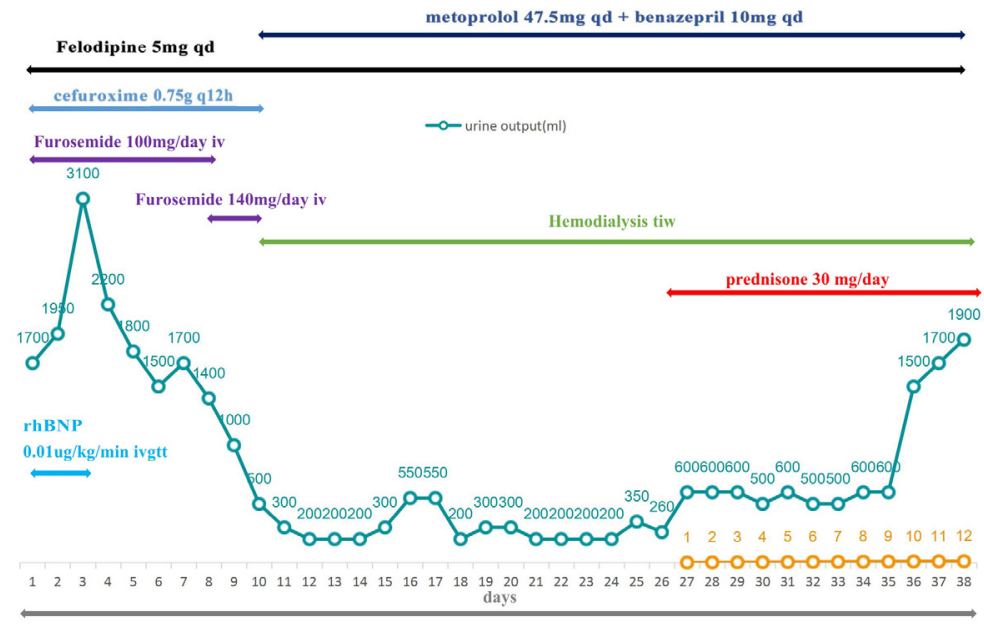

Figure 3. Treatment and prognosis of the patient. Prednisone $30 \mathrm{mg} / \mathrm{day}(0.5 \mathrm{mg} / \mathrm{kg})$ was administered for 14 days. After 2 weeks, the dosage was reduced to $20 \mathrm{mg} /$ day for 7 days; after 1 week, the dosage was reduced to $10 \mathrm{mg} /$ day for 7 days, and then the drug was stopped. Following $24 \mathrm{~h}$ of prednisone treatment, the patient's urine output reached $600 \mathrm{ml} /$ day. On the 12th day following treatment, the 24-h urine output reached 1,900 ml/day (without diuretics). At present, the patient's daily urine output can be stabilized at 1,500-2,000 ml. 
It should be emphasized again that the treatment administered to the patient in the present case lacks evidence. In fact, as far as is known, the case presented herein is the only case report currently focused on steroid therapy for advanced DKD. In order to provide a more substantial clinical basis for the treatment of such patients, further similar cases need to be examined and reviewed.

In conclusion, as demonstrated in the present study, if there is a typical manifestation of AKI in the course of the disease, even in patients with advanced kidney disease, the causes need to be carefully analyzed in order to identify and eliminate the factors leading to the deterioration of renal function. If AKI is clearly caused by AIN, it may then be possible that such patients may benefit from the use of steroids, even those who have developed severe renal fibrosis.

\section{Acknowledgements}

Not applicable.

\section{Funding}

No funding was received.

\section{Availability of data and materials}

The datasets used and/or analyzed during the current study are available from the corresponding author on reasonable request.

\section{Authors' contributions}

$\mathrm{JH}, \mathrm{SC}$ and $\mathrm{WH}$ conceived the case report, wrote the initial manuscript, and reviewed the final manuscript. JH, SC and $\mathrm{WH}$ interpreted and created the pathological images and reviewed the final manuscript. JH, SC and WH confirm the authenticity of all the raw data. All authors have read and approved the final manuscript.

\section{Ethics approval and consent to participate}

The patient provided informed consent for his involvement in the present study.

\section{Patient consent for publication}

The patient provided informed consent for the publishing of all the relevant clinical data.

\section{Competing interests}

The authors declare that they have no competing interests.

\section{References}

1. Zhou B, Lu Y, Hajifathalian K, Bentham J, Di Cesare M, Danaei G, Bixby H, Cowan MJ, Ali MK, Taddei C, et al; NCD Risk Factor Collaboration (NCD-RisC): Worldwide trends in diabetes since 1980: A pooled analysis of 751 population-based studies with 4.4 million participants. Lancet 387: 1513-1530, 2016.

2. Mann JF, Ørsted DD, Brown-Frandsen K, Marso SP, Poulter NR, Rasmussen S, Tornøe K, Zinman B and Buse JB; LEADER Steering Committee and Investigators: Liraglutide and renal outcomes in type 2 diabetes. N Engl J Med 377: 839-848, 2017.
3. Molitch ME, Adler AI,Flyvbjerg A, Nelson RG, So WY, Wanner C, Kasiske BL, Wheeler DC, de Zeeuw D and Mogensen CE: Diabetic kidney disease: A clinical update from Kidney Disease: Improving Global Outcomes. Kidney Int 87: 20-30, 2015.

4. Haas M, Spargo BH, Wit EJ and Meehan SM: Etiologies and outcome of acute renal insufficiency in older adults: A renal biopsy study of 259 cases. Am J Kidney Dis 35: 433-447, 2000.

5. Farrington K, Levison DA, Greenwood RN, Cattell WR and Baker LR: Renal biopsy in patients with unexplained renal impairment and normal kidney size. Q J Med 70: 221-233, 1989.

6. Perazella MA and Markowitz GS: Drug-induced acute interstitial nephritis. Nat Rev Nephrol 6: 461-470, 2010.

7. Praga M and González E: Acute interstitial nephritis. Kidney Int 77: 956-961, 2010.

8. Clarkson MR, Giblin L, O'Connell FP, O'Kelly P, Walshe JJ, Conlon P, O'Meara Y, Dormon A, Campbell E and Donohoe J: Acute interstitial nephritis: Clinical features and response to corticosteroid therapy. Nephrol Dial Transplant 19: 2778-2783, 2004.

9. González E, Gutiérrez E, Galeano C, Chevia C, de Sequera P, Bernis C, Parra EG, Delgado R, Sanz M, Ortiz M, et al; Grupo Madrileño De Nefritis Intersticiales: Early steroid treatment improves the recovery of renal function in patients with drug-induced acute interstitial nephritis. Kidney Int 73: 940-946, 2008.

10. Shinzato T, Ohara K, Kaminaga H, Sugase T, Masuda T, Nagata D, Saki K, Kinoshita Y, Kubo T, Shimizu T, et al: Acute interstitial nephritis and acute tubular injury due to a transdermal loxoprofen patch. Intern Med 59: 2733-2736, 2020.

11. Moledina DG and Perazella MA: Drug-induced acute interstitial nephritis. Clin J Am Soc Nephrol 12: 2046-2049, 2017.

12. Rangaswami J, Bhalla V, Blair JEA, Chang TI, Costa S, Lentine KL, Lerma EV, Mezue K, Molitch M, Mullens W, et al; American Heart Association Council on the Kidney in Cardiovascular Disease and Council on Clinical Cardiology: Cardiorenal Syndrome: Classification, pathophysiology, diagnosis, and treatment strategies: A scientific statement from the American Heart Association. Circulation 139: e840-e878, 2019.

13. Kalra S, Aydin H, Sahay M, Ghosh S, Ruder S, Tiwaskar M, Kilov G, Kishor K, Nair T, Makkar V, et al: Cardiorenal syndrome in type 2 diabetes mellitus - rational use of sodium-glucose cotransporter-2 inhibitors. Eur Endocrinol 16: 113-121, 2020.

14. Raghavan R and Eknoyan G: Acute interstitial nephritis - a reappraisal and update. Clin Nephrol 82: 149-162, 2014.

15. Neilson EG: Mechanisms of disease: Fibroblasts--a new look at an old problem. Nat Clin Pract Nephrol 2: 101-108, 2006.

16. Li T, Wilcox CS, Lipkowitz MS, Gordon-Cappitelli J and Dragoi S: Rationale and strategies for preserving residual kidney function in dialysis patients. Am J Nephrol 50: 411-421, 2019.

17. Termorshuizen F, Korevaar JC, Dekker FW, van Manen JG, Boeschoten EW and Krediet RT; NECOSAD Study Group: The relative importance of residual renal function compared with peritoneal clearance for patient survival and quality of life: An analysis of the Netherlands Cooperative Study on the Adequacy of Dialysis (NECOSAD)-2. Am J Kidney Dis 41: 1293-1302, 2003.

18. Bargman JM, Thorpe KE and Churchill DN: Relative contribution of residual renal function and peritoneal clearance to adequacy of dialysis: A reanalysis of the CANUSA study. J Am Soc Nephrol 12: 2158-2162, 2001.

19. Obi Y, Streja E, Rhee CM, Ravel V, Amin AN, Cupisti A Chen J, Mathew AT, Kovesdy CP, Mehrotra R, et al: Incremental Hemodialysis, Residual Kidney Function, and Mortality Risk in Incident Dialysis Patients: A Cohort Study. Am J Kidney Dis 68: 256-265, 2016.

20. Shafi T, Jaar BG, Plantinga LC, Fink NE, Sadler JH, Parekh RS, Powe NR and Coresh J: Association of residual urine output with mortality, quality of life, and inflammation in incident hemodialysis patients: The Choices for Healthy Outcomes in Caring for End-Stage Renal Disease (CHOICE) Study. Am J Kidney Dis 56: 348-358, 2010.

21. Kritmetapak K, Anutrakulchai S, Pongchaiyakul C and Puapairoj A: Clinical and pathological characteristics of non-diabetic renal disease in type 2 diabetes patients. Clin Kidney J 11: 342-347, 2018.

This work is licensed under a Creative Commons Attribution 4.0 International (CC BY 4.0) License. 